\title{
Long time existence results for solutions of water waves equations*
}

\author{
Jean-Marc Delort \\ Université Paris 13, \\ Sorbonne Paris Cité, LAGA, CNRS (UMR 7539), \\ 99, Avenue J.-B. Clément, \\ F-93430 Villetaneuse
}

\begin{abstract}
We present in this report various results obtained during the last years by several authors about the problem of long time existence of solutions of water waves and related equations with initial data that are small, smooth, and decaying at infinity. After recalling some facts about local existence theory, we focus mainly on global existence theorems for gravity waves equations proved by Ionescu-Pusateri [35, Alazard-Delort [6, 7] and Ifrim-Tataru 32]. We describe some of the ideas of the proofs of these theorems, and conclude the paper mentioning related results.
\end{abstract}

\section{The water waves equations}

Consider an incompressible and irrotational fluid, of constant density equal to one, in a vertical gravity field of intensity $g$. Assume that at time $t$, the domain occupied by the fluid is

$$
\Omega_{t}=\left\{(x, z) \in \mathbb{R}^{d} \times \mathbb{R} ;-H_{0}<z<\eta(t, x)\right\},
$$

where $\eta(t, \cdot): \mathbb{R}^{d} \rightarrow \mathbb{R}$ is such that $\inf _{x \in \mathbb{R}^{d}} \eta(t, x)>-H_{0}$, and either $H_{0} \in$ ]0, $+\infty$ [ (for a fluid of finite depth) or $H_{0}=+\infty$ (for an infinite depth fluid).

The velocity $U$ in the fluid solves in $\Omega=\left\{(t, x) ; x \in \Omega_{t}\right\}$ the incompressible Euler equations

$$
\begin{aligned}
\partial_{t} U+U \cdot \nabla_{x, z} U & =-\nabla_{x, z} p-g e_{z} \\
\operatorname{div} U & =0
\end{aligned}
$$

*From joint work with Thomas Alazard. MR Subjects Classification: 76B15, 35Q35, 35B40, 35S50. Partially supported by the ANR project 13-BS01-0010-02 "Analyse asymptotique des équations aux dérivées partielles d'évolution". 
where $e_{z}$ is the vertical unit vector and $p$ the pressure inside the fluid. Moreover, the normal velocity at the bottom satisfies $\left.U \cdot e_{z}\right|_{z=-H_{0}}=0$ (when $H_{0}<+\infty$ ) or $U \rightarrow 0$ when $z$ goes to $-\infty$ (if $H_{0}=-\infty$ ). Finally, the free surface is driven by the velocity of the fluid at each point of the interface $z=\eta(t, x)$, which is translated by

$$
\partial_{t} \eta(t, x)=\left.\sqrt{1+\left|\nabla_{x} \eta(t, x)\right|^{2}} U(t, x, z) \cdot n\right|_{z=\eta(t, x)},
$$

where $n$ is the unit outward normal vector to $\Omega_{t}$ at $(x, \eta(t, x))$. Moreover, the pressure above the fluid is equal to the constant atmospheric pressure, that we may take equal to zero. At the interface $z=\eta(t, x)$, the pressure of the fluid will be given by

$$
\left.p\right|_{z=\eta(t, x)}=-\kappa \operatorname{div}\left(\frac{\nabla_{x} \eta}{\sqrt{1+\left|\nabla_{x} \eta\right|^{2}}}\right),
$$

where the constant $\kappa \geq 0$ is the surface tension. Since, as the fluid is also assumed to be irrotational, $\operatorname{curl} U=0$, one may express the velocity $U$ from a potential $\Phi$ by $U=\nabla_{(x, z)} \Phi$. The incompressibility implies $\Delta_{(x, z)} \Phi=0$ and the Euler equation (1) allows one to write an equation for $\Phi$ :

$$
\partial_{t} \Phi+\frac{1}{2}\left|\nabla_{(x, z)} \Phi\right|^{2}+g z=-p .
$$

Moreover, one has the boundary condition at the bottom

$$
\begin{aligned}
& \left.\partial_{z} \Phi\right|_{z=-H_{0}}=0 \text { (for finite depth) } \\
& \nabla_{(x, z)} \Phi \rightarrow 0 \text { if } z \rightarrow-\infty \text { (for infinite depth) }
\end{aligned}
$$

and, expressing $U=\nabla_{(x, z)} \Phi$ in $(2)$, one gets

$$
\partial_{t} \eta(t, x)=\sqrt{1+\left|\nabla_{x} \eta(t, x)\right|^{2}} \partial_{n} \Phi(t, x, z) \text { on } z=\eta(t, x),
$$

denoting by $\partial_{n}$ the outwards normal unit derivative at the free interface. The Craig-Sulem-Zakharov formulation of the water waves system, given in [59] and [20] (see also the book of Lannes [39]) is obtained expressing in (4), (6), the potential $\Phi$ from its boundary data. More precisely, denote by $\psi$ the restriction of $\Phi$ to the interface $z=\eta(t, x)$. Then $\Phi$ solves the elliptic boundary values problem

$$
\begin{aligned}
\Delta_{(x, z)} \Phi & =0 \\
\left.\Phi\right|_{z=\eta(t, x)} & =\psi \\
\left.\partial_{z} \Phi\right|_{z=-H_{0}} & =0
\end{aligned}
$$

(or, for the last condition, $\nabla_{(x, z)} \Phi \rightarrow 0$ when $z \rightarrow-\infty$ in the case of infinite depth). One denotes by $G(\eta) \psi$ the Dirichlet-Neuman operator defined by

$$
G(\eta) \psi=\left.\sqrt{1+\left|\nabla_{x} \eta\right|^{2}} \partial_{n} \Phi\right|_{z=\eta(t, x)},
$$


where $\Phi$ solves (7). Plugging this information in (4) restricted to $z=\eta(t, x)$, (6), and using (3), one obtains for $(\eta, \psi)$ the system

$$
\begin{aligned}
& \partial_{t} \eta=G(\eta) \psi \\
& \partial_{t} \psi=-g \eta-\frac{1}{2}\left|\nabla_{x} \psi\right|^{2}+\frac{\left(G(\eta) \psi+\nabla_{x} \eta \cdot \nabla_{x} \psi\right)^{2}}{2\left(1+\left|\nabla_{x} \eta\right|^{2}\right)}+\kappa \operatorname{div}\left(\frac{\nabla_{x} \eta}{\sqrt{1+\left|\nabla_{x} \eta\right|^{2}}}\right) .
\end{aligned}
$$

This is the system we intend to study below, in the case of pure gravity water waves, i.e. when $g>0$ and $\kappa=0$.

\section{The question of local existence}

The question of existence of local in time solutions for system (9) (when $\kappa=0$ and the fluid depth is infinite) with data in Sobolev spaces remained open for a long time, and was fully answered in 1997 by Sijue Wu in the seminal paper [54] when $x$ belongs to $\mathbb{R}$ and in [55] when $x$ is in $\mathbb{R}^{2}$. As in the subsequent sections we shall be interested mainly in the one dimensional problem, we assume for the rest of this section that the space variable $x$ belongs to $\mathbb{R}$. The difficulty in order to prove local existence may be seen in the following way: if one writes (9) under the form

$$
\partial_{t}\left[\begin{array}{l}
\eta \\
\psi
\end{array}\right]=A(\eta, \psi)\left[\begin{array}{l}
\eta \\
\psi
\end{array}\right],
$$

where $A$ is a pseudo-differential operator with coefficients with limited smoothness, defined by

$$
A(\eta, \psi)\left[\begin{array}{c}
\dot{\eta} \\
\dot{\psi}
\end{array}\right]=\frac{1}{2 \pi} \int e^{i x \xi} M(x, \xi)\left[\begin{array}{l}
\hat{\eta}(t, \xi) \\
\hat{\psi}(t, \xi)
\end{array}\right] d \xi,
$$

then the matrix symbol $M(x, \xi)$ (that depends on $(\eta, \psi))$ has eigenvalues whose real part may go to infinity with $|\xi|$. This instability prevents one from getting energy inequalities.

A way to overcome this difficulty, and to prove local existence for a restricted class of energy data, has been introduced by Nalimov [41] (for infinite depth fluids) and Yosihara [58, for finite depth ones. See also the work of Craig [19]. The local existence of solutions for arbitrary Sobolev initial data has been established for infinite depth fluids by Sijue $\mathrm{Wu}$ [54, 55]. Actually, her work is not limited to an interface given by a graph $z=\eta(t, x)$, but allows upper boundaries for $\Omega_{t}$ given by any non self-intersecting smooth curve. The method used by the above authors was relying on the Lagrangian formulation of the water waves system, and has been at the origin of a lot of works concerning related models (like for instance the capillary-gravity wave equations, where $\kappa$ in (9) is non zero) with finite or infinite depth, both for localized or unlocalized initial data. We cite in particular results of local existence of Ambrose [11, Ambrose-Masmoudi [12, Coutand-Shkoller [18. At the same time, a more geometric approach to study free boundary value problems has been developed by 
Christodoulo-Lindblad [17], Lindblad [40] and in a series of papers of Shatah and Zhang [45, 46, 47.

On the other hand, Lannes [37] introduced an Eulerian approach to local existence, expressing the problem in terms of a "good unknown" $\omega$ instead of $\psi$. Such a "good unknown" had been introduced in the framework of free boundary problems by Alinhac in [9, 10. For water waves equations (in any dimension, and with eventually a bottom), Lannes showed that the system, written in terms of $(\eta, \omega)$, is a quasi-linear hyperbolic equation, for which Sobolev energy estimates are available and provide local existence of solutions.

This new unknown was later implemented by Alazard and Métivier 8 in a paradifferential framework. Let us describe how it may be defined for problem (9) in one space dimension. Recall that the paraproduct $T_{a} b$ [16] of a bounded function $a$ and a tempered distribution $b$ may be defined by

$$
\widehat{T_{a} b}(\xi)=\int_{\xi_{1}+\xi_{2}=\xi} \chi\left(\xi_{1}, \xi_{2}\right) \hat{a}\left(\xi_{1}\right) \hat{b}\left(\xi_{2}\right) d \xi_{1} d \xi_{2},
$$

where $\chi$ is a smooth function satisfying

$$
\left|\partial_{\xi_{1}}^{\alpha_{1}} \partial_{\xi_{2}}^{\alpha_{2}} \chi\left(\xi_{1}, \xi_{2}\right)\right| \leq C\left(1+\left|\xi_{1}\right|+\left|\xi_{2}\right|\right)^{-\alpha_{1}-\alpha_{2}},
$$

supported for $\left|\xi_{1}\right| \leq\left(1+\left|\xi_{2}\right|\right) / 10$, equal to one for $\left|\xi_{1}\right| \leq\left(1+\left|\xi_{2}\right|\right) / 100$ for instance. Then if $a$ is in $L^{\infty}$ and $b$ is in a Sobolev space $H^{s}$, the paraproduct $T_{a} b$ belongs to $H^{s}$, whatever the value of $s$. Let us introduce:

Definition 1 For $\eta$ in $H^{s}(\mathbb{R}), \psi$ such that $\left|D_{x}\right|^{\frac{1}{2}} \psi \in H^{s}(\mathbb{R})$, with s large enough, set

$$
B(\eta) \psi \stackrel{\text { def }}{=} \frac{G(\eta) \psi+\left(\partial_{x} \eta\right)\left(\partial_{x} \psi\right)}{1+\left(\partial_{x} \eta\right)^{2}} .
$$

One defines the good unknown $\omega$ by

$$
\omega=\psi-T_{B(\eta) \psi} \eta .
$$

Using this good unknown, and a paralinearization of the Dirichlet-Neuman operator due to Alazard and Métivier [8], Alazard, Burq and Zuily [4, 5, 2, 3] proved local existence theorems for (9) (with or without surface tension) under weaker regularity assumptions on the Cauchy data than in previous works.

Finally, let us mention a last approach to local existence theory, encompassing in some way the Lagrangian formulation and the use of a good unknown, proposed by Hunter, Ifrim and Tataru [28, that relies on the introduction of new quantities defined as boundary values of holomorphic functions.

\section{Global existence with small decaying data}

Once local existence of solutions to system (9) is established, it is natural to ask the question of long time existence for small smooth enough initial data 
that have some decay at infinity. Up to the last but one section, we discuss this problem for 9 in infinite depth, when the surface tension $\kappa$ is equal to zero, and space dimension $d$ is equal to one or two, postponing to the last section references to other models (finite depth, presence of surface tension terms, etc).

One checks easily that the solution of (9) with $\kappa=0$ linearized on the zero solution, with decaying initial data, has $L^{\infty}$ norm that is $O\left(t^{-\frac{d}{2}}\right)$ when $t$ goes to infinity, in $d$ space dimension, because of the dispersive effect. The first breakthrough concerning long time existence of solutions is due to Sijue $\mathrm{Wu}$ [56] who proved that, in one space dimension, for smooth decaying Cauchy data of small size $\epsilon$, the solution exists over a time interval of length $e^{c / \epsilon}$ for some positive constant $c$. For two space dimensions, the stronger decay rate of solutions of the linearized equation makes expect better results. Actually $\mathrm{Wu}$ [57] and Germain, Masmoudi and Shatah [26] proved that then solutions are global if the data are smooth, small, and decaying enough. Moreover, there is scattering [26], i.e. the solutions of the nonlinear problem have the same asymptotics as solutions of the linearized equation on the zero state when time goes to infinity.

The main result we report on here concerns, in one space dimension, global existence of solutions for small, smooth, decaying Cauchy data, and modified scattering. This result has been obtained independently by Ionescu-Pusateri [35], using a combination of the Lagrangian and the Eulerian formulations of the equations, and by Alazard-Delort [6, 7], through the Eulerian formulation and the good unknown introduced above. An alternative approach, based on the "holomorphic coordinates" of Hunter, Ifrim and Tataru, has also been proposed by Ifrim and Tataru 32 .

We state below the result of 6 . We compare it next with the statements of [35] and 32.

Theorem 2 Fix $\gamma \in \mathbb{R}-\frac{1}{2} \mathbb{N}$ a large enough number, $s, s_{1}$ in $\mathbb{N}$ such that $s_{1} \geq \frac{s}{2}+\gamma$ and $s-s_{1}$ is large enough. There is $\epsilon_{0}>0$ and for any $\left.\epsilon \in\right] 0, \epsilon_{0}[$, any couple $\left(\eta_{0}, \psi_{0}\right)$ of functions satisfying for any integer $0 \leq p \leq s_{1}$,

$$
\begin{array}{r}
\left(x \partial_{x}\right)^{p} \eta_{0} \in H^{s-p}(\mathbb{R}), \quad\left|D_{x}\right|^{\frac{1}{2}}\left(x \partial_{x}\right)^{p} \psi_{0} \in H^{s-p-\frac{1}{2}}(\mathbb{R}) \\
\left|D_{x}\right|^{\frac{1}{2}}\left(x \partial_{x}\right)^{p}\left[\psi_{0}-T_{B\left(\eta_{0}\right) \psi_{0}} \eta_{0}\right] \in H^{s-p}(\mathbb{R}),
\end{array}
$$

with the norms in the above spaces smaller or equal to one, the evolution problem (9) (in one space dimension, with $\kappa=0$ and infinite depth) with initial data $\left.\psi\right|_{t=1}=\epsilon \psi_{0},\left.\eta\right|_{t=1}=\epsilon \eta_{0}$, has a unique solution, continuous with values in the set of functions satisfying (14), defined on the whole interval $[1,+\infty[$. Moreover, if we define $u=\left|D_{x}\right|^{\frac{1}{2}} \psi+i \eta$, we have the following asymptotics when $t$ goes to infinity:

$$
u(t, x)=\frac{\epsilon}{\sqrt{t}} \alpha_{\epsilon}\left(\frac{x}{t}\right) \exp \left[\frac{i t}{4|x / t|}+\frac{i \epsilon^{2}}{64} \frac{\left|\alpha_{\epsilon}(x / t)\right|^{2}}{|x / t|^{5}} \log t\right]+\epsilon t^{-\frac{1}{2}-\theta} \rho(t, x),
$$

where $\left(\alpha_{\epsilon}\right)_{\epsilon \in] 0,1]}$ is a bounded family of functions of $C(\mathbb{R}) \cap L^{\infty}(\mathbb{R}), \theta$ is positive and $\|\rho(t, \cdot)\|_{L^{\infty}}$ is bounded for $t \geq 1$. 
Remark: The asymptotics (15) show that the global solution displays a modified scattering, where the phase of oscillation of linear solutions is modulated by an extra logarithmic term. This term, that becomes significant for times $t$ such that $\log t \gg \epsilon^{-2}$, is responsible of the new difficulties arising in one dimensional problems versus two dimensional ones.

Let us compare the above result with the ones of Ionescu-Pusateri [35] and Ifrim-Tataru 32 .

In the first of these references, a similar result of global existence is obtained under a weaker decay condition on the initial data, namely conditions of the form (14) have to be imposed only for $0 \leq p \leq 1$. Moreover, one assumes the smallness of a " $Z$-norm", that controls $\left\||\xi|^{\beta} \hat{u}_{0}(\xi) \mathbb{1}_{|\xi| \leq 1}\right\|_{L^{\infty}}$, for some $\beta>0$, where $u_{0}=\left|D_{x}\right|^{\frac{1}{2}} \psi_{0}+i \eta_{0}$. This norm plays a key role in the proof of the optimal decay of the solution and of the modified scattering.

The result of Ifrim and Tataru is expressed from slightly different unknowns than $(\eta, \psi)$ above. Actually, these authors introduce $Z$, a parameterization of the boundary, chosen in such a way that it is the boundary value of some holomorphic function in the fluid domain, and $Q$, the boundary value of another holomorphic function in the fluid, whose real part coincides with the velocity potential at the boundary. They assume that a Sobolev norm of $\left.(Z(t, \alpha)-\alpha)\right|_{t=0}$ and $\left.Q(t, \alpha)\right|_{t=0}$, involving essentially at most six derivatives, is small and that an $H^{1}$ norm of the action of $x \partial_{x}$ on an expression of these quantities is also small at the initial time. They obtain then global existence and modified scattering.

The proofs of the results of global existence of [6, 32, 35] might differ in their technical details, but the difficulties that have to be overcome are essentially the same. In the rest of that report, we shall try to describe them in a non technical way, using most of the time some simplified toy models instead of the full equation (9). We shall use the formulation of the equation in terms of $\eta$ and the good unknown $\omega$, following [6, 7], but will make also frequent references to the works [35, 32].

\section{Quadratic terms. Normal forms}

Consider first a model equation of the form

$$
\begin{aligned}
\left(D_{t}-p\left(D_{x}\right)\right) u & =N(u) \\
\left.u\right|_{t=1} & =\epsilon u_{0}
\end{aligned}
$$

where $D_{t}=\frac{1}{i} \frac{\partial}{\partial t}, D_{x}=\frac{1}{i} \frac{\partial}{\partial x}, p(\xi)$ is a real valued elliptic Fourier multiplier and $N(u)$ is a nonlinearity vanishing at least at order two at zero. The water wave system, linearized at the zero state, may be expressed in terms of $u=$ $\left|D_{x}\right|^{\frac{1}{2}} \psi+i \eta$, as $\left(D_{t}-\left|D_{x}\right|^{\frac{1}{2}}\right) u=0$, so that 16 is a model for that system if we take $p(\xi)=|\xi|^{\frac{1}{2}}$.

Assume first that $N$ is semi-linear and at least cubic at zero, in that sense that it satisfies an estimate

$$
\|N(u)\|_{H^{s}} \leq C\|u\|_{L^{\infty}}^{2}\|u\|_{H^{s}}
$$


for any $s>0$ for instance. Then the Sobolev energy inequality associated to (16) writes

$$
\|u(t, \cdot)\|_{H^{s}} \leq\|u(1, \cdot)\|_{H^{s}}+C \int_{1}^{t}\|u(\tau, \cdot)\|_{L^{\infty}}^{2}\|u(\tau, \cdot)\|_{H^{s}} d \tau .
$$

If one assumes that, in addition, one has some a priori estimate for $\|u(t, \cdot)\|_{L^{\infty}} \leq$ $B \epsilon t^{-\frac{1}{2}}$ (as the one we expect according to 15 ), we deduce by Gronwall inequality a bound

$$
\|u(t, \cdot)\|_{H^{s}} \leq C\|u(0, \cdot)\|_{H^{s}} t^{C B^{2} \epsilon^{2}}
$$

i.e. a control of the Sobolev norm that is not uniform, but given in terms of an arbitrary small power of $t$ (if $\epsilon$ is small). As we shall see, a bound of this type will be sufficient for our goals.

On the other hand, in the system (9) we are really interested in, the nonlinearity is quadratic, and not cubic. For the toy model (16), this would mean assuming, instead of (17), $\|N(u)\|_{H^{s}} \leq C\|u\|_{L^{\infty}}\|u\|_{H^{s}}$, so that (19) would be replaced by

$$
\|u(t, \cdot)\|_{H^{s}} \leq C\|u(0, \cdot)\|_{H^{s}} \exp [C B \epsilon \sqrt{t}]
$$

which is useless if one wants to study solutions on time intervals of length larger than $\epsilon^{-2}$. The way to actually obtain estimates of the form $\left.\sqrt{19}\right)$, including for a quadratic nonlinearity, is well known in the semi-linear case: this is the normal forms method of Shatah [44, that allows to reduce a quadratic nonlinearity to a cubic one. (We refer also to the more recent developments of that method introduced in the work of Germain, Masmoudi and Shatah [25. See also the Bourbaki seminar of Lannes [38] and references therein.) For quadratic nonlinearities, $N(u)=u^{2}$ for instance, the idea of the method is to look for a new unknown $\phi=u+E(u, u)$, where $E(u, u)$ is a quadratic expression of the form

$$
E(u, u)=\frac{1}{2 \pi} \int e^{i x\left(\xi_{1}+\xi_{2}\right)} m\left(\xi_{1}, \xi_{2}\right) \hat{u}\left(\xi_{1}\right) \hat{u}\left(\xi_{2}\right) d \xi_{1} d \xi_{2},
$$

chosen in such a way that

$$
\left(D_{t}-p\left(D_{x}\right)\right) \phi=\tilde{N}(\phi)
$$

for a new nonlinearity $\tilde{N}$ vanishing at third order at zero. A direct computation using (16) shows that one has to take

$$
m\left(\xi_{1}, \xi_{2}\right)=\left(p\left(\xi_{1}\right)+p\left(\xi_{2}\right)-p\left(\xi_{1}+\xi_{2}\right)\right)^{-1}
$$

in order to achieve that. The transformation $u \rightarrow \phi$ will then be bounded on $H^{s}$ spaces if, for large frequencies $\xi_{1}, \xi_{2}$, one has a bound of the form

$$
\left|m\left(\xi_{1}, \xi_{2}\right)\right| \leq C \min \left(\left|\xi_{1}\right|,\left|\xi_{2}\right|\right)^{N_{0}}
$$

for some fixed $N_{0}$. In that way, if $s$ is large enough relatively to $N_{0},\|E(u, u)\|_{H^{s}} \leq$ $C\|u\|_{H^{s}}^{2}$, which shows that $u \rightarrow \phi$ is a diffeomorphism from a neighborhood of 
zero to its image. For the toy model 16 with $p(\xi)=|\xi|^{\frac{1}{2}}$, it is easy to see that an estimate of the form (23) holds for large frequencies. For small ones, a degeneracy happens, but, in the case of the water waves system (9), it will be compensated by the fact that in the nonlinearity, operators whose symbols vanish at $\xi=0$ act on $u$.

In the case of the water waves system (9), one would like to perform as well a similar normal forms method in order to eliminate quadratic terms. The difficulty is that, 9 being quasi-linear, 16 is not a convincing model for it, as the nonlinearity there depends only on $u$, and not on first order derivatives of $u$. On the other hand, if one replaces $N(u)$ by a quadratic term of the form $u D_{x} u$, and tries to eliminate it looking for a new unknown $\phi=u+E(u, u)$, one would have to express $E$ by $(20)$, but with a symbol $m\left(\xi_{1}, \xi_{2}\right)$ given by

$$
m\left(\xi_{1}, \xi_{2}\right)=\left(p\left(\xi_{1}\right)+p\left(\xi_{2}\right)-p\left(\xi_{1}+\xi_{2}\right)\right)^{-1} \xi_{2}
$$

which loses one derivative, so that $\|\phi\|_{H^{s}}$ is only estimated from $\|u\|_{H^{s+1}}$. A way to circumvent that difficulty, that appears already in the work of Ozawa, Tsutaya and Tsutsumi [42, is to try to combine the normal forms construction with the idea used to get quasi-linear energy inequalities. In order to explain that, we have to make appeal to a more accurate model than $(16)$. We have seen in section 2 that, in order to avoid losses of derivatives in energy estimates, it is convenient to write the water waves equation in terms of the unknowns $\left(\eta, \omega=\psi-T_{B(\eta) \psi} \eta\right)$. More precisely, if one introduces $U=\left[\begin{array}{c}\eta+T_{\alpha} \eta \\ \left|D_{x}\right|^{\frac{1}{2}} \omega\end{array}\right]$, where $\alpha$ is some explicit function of $u=\left|D_{x}\right|^{\frac{1}{2}} \psi+i \eta$, vanishing at $u=0$, one may write system (9) under the form

$$
\partial_{t} U+\left[\begin{array}{cc}
0 & -\left|D_{x}\right|^{\frac{1}{2}} \\
\left|D_{x}\right|^{\frac{1}{2}} & 0
\end{array}\right] U+Q(u) U+S(u) U+C(u) U=G
$$

where we used the following notation:

- The right hand side $G$ is a semi-linear cubic term. This means that it will satisfy for $s \gg \rho \gg 1$ estimates of the following form:

$$
\|G\|_{H^{s}} \leq C\left(\|u\|_{C^{\rho}}\right)\|u\|_{C^{\rho}}^{2}\|U\|_{H^{s}}
$$

Such a term satisfies thus bounds of the form (17) (with the $L^{\infty}$ norm replaced by a Hölder norm). If we write the Sobolev energy inequality associated to (24), and forget the contributions of $Q, S, C$, we would thus get

$$
\|U(t, \cdot)\|_{H^{s}} \leq\|U(t, \cdot)\|_{H^{s}}+C \int_{1}^{t}\|u(\tau, \cdot)\|_{C^{\rho}}^{2}\|U(\tau, \cdot)\|_{H^{s}} d \tau .
$$

Combined with an a priori bound $\|u(\tau, \cdot)\|_{C^{\rho}}=O\left(\epsilon t^{-\frac{1}{2}}\right)$, this would give for $\|U(t, \cdot)\|_{H^{s}}$ an estimate of the form 19 .

- The term $C(u) U$ is a cubic contribution given in terms of a paradifferential operator of order one $C(u)$ : this means that

$$
C(u) v=\frac{1}{2 \pi} \int e^{i x \xi} c(u ; x, \xi) \hat{v}(\xi) d \xi
$$


where $u \rightarrow c(u ; x, \xi)$ is a quadratic map with values in the set of functions of $(x, \xi)$ satisfying bounds of the form

$$
\left|\partial_{\xi}^{\beta} c(u ; x, \xi)\right| \leq C\left(\|u\|_{C^{\rho}}\right)\|u\|_{C^{\rho}}^{2}\langle\xi\rangle^{1-\beta}
$$

for some fixed $\rho>0$ independent of $\beta$, and that moreover the Fourier transform with respect to $x$ of $x \rightarrow c(u ; x, \xi)$, that we denote by $\hat{c}(u ; \eta, \xi)$, is supported for $|\eta| \ll|\xi|$. The paraproduct recalled in section 3 is a special case of the above definition, corresponding to the case when $c(u ; x, \xi)$ does not depend on $\xi$. When one computes the time derivative

$$
\partial_{t}\|U(t, \cdot)\|_{L^{2}}^{2}=2 \operatorname{Re}\left\langle\partial_{t} U, U\right\rangle,
$$

the contribution to the right hand side coming from the $C(u) U$ term in 24 may be written as

$$
\left\langle\left(C(u)+C(u)^{*}\right) U, U\right\rangle .
$$

Because of the explicit form of the operator $C(u)$, one may check that $C(u)+$ $C(u)^{*}$ is of order zero. (This reflects the fact that the equation (24) one reduced to using the good unknown is hyperbolic). Taking into account that operators of order zero are bounded on $L^{2}$, and that $u \rightarrow C(u)$ vanishes at order two at zero, one gets for (30) an upper bound in $\|u\|_{C \rho}^{2}\|U\|_{L^{2}}$. Since the same reasoning may be done replacing in $(29) L^{2}$ norms by Sobolev ones, we see that the term $C(u) U$ in (24) would also generate in an energy inequality a contribution bounded from above by the right hand side of $(26)$.

- The terms $Q(u) U$ and $S(u) U$ are quadratic contributions, with $Q(u)$ a paradifferential operator of order one, linear in $u$, and $S(u)$ a smoothing operator. These contributions have to be eliminated by normal forms. As $S(u) U$ is a semi-linear term, it may be eliminated essentially using a quadratic correction of the form (20), up to some technical issues that we do not discuss here. On the other hand, $Q(u) U$ is a quasi-linear contribution, and as we have seen above, a brutal elimination could give rise to an unbounded normal forms transformation. But again, as in (30), only the operator $Q(u)+Q(u)^{*}$ plays a role in an energy inequality, and by the hyperbolic structure of equation (24), such an operator is of order zero instead of one. Consequently, trying to eliminate only this term from the right hand side of (24), one may construct, including in this quasi-linear framework, a change of unknown $U \rightarrow \phi=U+E(u) U$, where $E$ is bounded on $H^{s}$, and such that $\phi$, and thus $U$, will obey an estimate of the form (26). One has thus reduced morally to a cubic nonlinearity.

We may summarize this in the following statement:

Proposition 3 There is a bounded linear map $U \rightarrow \phi=U+E(u) U$, going from a neighborhood of zero in $H^{s}$ to a neighborhood of zero in $H^{s}$, when $u$ is in a ball of $C^{\rho}$ and $1 \ll \rho \ll s$, that transforms equation (24) for $U$ into

$$
\partial_{t} \phi+\left[\begin{array}{cc}
0 & -\left|D_{x}\right|^{\frac{1}{2}} \\
\left|D_{x}\right|^{\frac{1}{2}} & 0
\end{array}\right] \phi+L(u) \phi+C(u) \phi=\Gamma,
$$


where $C(u)$ is as in (24), $\Gamma$ is a cubic semi-linear term, and $L(u)$ is linear in $u$ and satisfies $\operatorname{Re}\langle L(u) \phi, \phi\rangle_{H^{s}}=0$.

The normal forms method outlined above does not eliminate the whole quadratic part of the nonlinearity, but only those terms in it that give nonzero contributions to the energy. Because of that, one might think that it should be possible to perform the normal form procedure on the Sobolev energy itself instead of the equation. Such an approach has been performed by Hunter, Ifrim, Tataru and Wong [29] and used by Hunter-Ifrim-Tataru [28] in order to give another proof of the almost global existence result of Sijue Wu [56]. They applied next the same method, that does not require the paralinearization of the equation, in their proof of the global existence result [32]. Notice that similar ideas, (combined with an a priori paralinearization) are used in [21, 22, 23 . for quasi-linear KleinGordon equations on some compact manifolds. Such an approach is particularly convenient when one studies a Hamiltonian system and wants to keep track of the Hamiltonian structure all along a normal forms reduction procedure.

On the other hand, the elimination of the contributions of the quadratic part of the nonlinearity to the energy, as a first step towards the proof of a global existence result, is performed by Ionescu and Pusateri [35] using the transformation in Lagrangian coordinates of $\mathrm{Wu}$ [56], Totz and $\mathrm{Wu}$ [49].

\section{Global existence: bootstrap procedure}

We shall discuss from now on the proof of the global existence result of Theorem 2 on a model equation. If $p(\xi)$ is some elliptic Fourier multiplier, consider

$$
\left(D_{t}-p\left(D_{x}\right)\right) u=N(u)
$$

with $N(u)$ a cubic nonlinearity of the form

$$
N(u)=\alpha_{3} u^{3}+\alpha_{1}|u|^{2} u+\alpha_{-1}|u|^{2} \bar{u}+\alpha_{-3} \bar{u}^{3},
$$

where $\alpha_{j}$ are complex numbers, with $\alpha_{1}$ real. Of course, equation 32 is a simplification of the real system we are studying, but it is a convincing prototype of the problem after the normal forms procedure of the preceding section has been performed in order to reduce to a cubic nonlinearity. The fact that it is semilinear instead of quasilinear just brings some inessential technical simplifications at this level. Let us introduce the Klainerman vector field

$$
Z=t D_{t}+2 x D_{x}
$$

that satisfies when $p(\xi)=|\xi|^{\frac{1}{2}}$

$$
\left[D_{t}-p\left(D_{x}\right), Z\right]=D_{t}-p\left(D_{x}\right)
$$

so that $Z u$ solves

$$
\left(D_{t}-p\left(D_{x}\right)\right)(Z u)=N(u)+Z N(u) .
$$


The key of global existence (and modified scattering) is the proof of the following bootstrap assertion:

Proposition 4 There are positive constants $A, B, K, s_{0}$ and $\left.\left.\epsilon_{0} \in\right] 0,1\right]$ such that, for any $s \geq s_{0}$, any $\left.\epsilon \in\right] 0, \epsilon_{0}\left[\right.$, any $u_{0}$ in $H^{s}(\mathbb{R})$ satisfying

$$
\left\|u_{0}\right\|_{H^{s}}+\left\|x \partial_{x} u_{0}\right\|_{L^{2}} \leq \epsilon,
$$

for any solution $u$ of (32) with initial $u_{0}$ that exists over some interval $I=[1, T]$, and satisfies for any $t$ in $I$,

$$
\begin{aligned}
& \text { (A) }\|u(t, \cdot)\|_{H^{s}}+\|Z u(t, \cdot)\|_{L^{2}} \leq A \epsilon t^{K \epsilon^{2}} \\
& \text { (B) }\|u(t, \cdot)\|_{L^{\infty}} \leq \frac{B \epsilon}{\sqrt{t}}
\end{aligned}
$$

then, for $t$ in the same interval I, one has actually

$$
\begin{array}{ll}
\left(A^{\prime}\right) & \|u(t, \cdot)\|_{H^{s}}+\|Z u(t, \cdot)\|_{L^{2}} \leq \frac{A}{2} \epsilon t^{K \epsilon^{2}} \\
\left(B^{\prime}\right) & \|u(t, \cdot)\|_{L^{\infty}} \leq \frac{B \epsilon}{2 \sqrt{t}}
\end{array}
$$

Remarks: • In the water waves system we are interested in, the quasi-linear nature of the problem makes that one has to control some derivatives of $Z u$ in $L^{2}$ and of $u$ in $L^{\infty}$, i.e. one has to replace in $(A),\left(A^{\prime}\right),\|Z u(t, \cdot)\|_{L^{2}}$ by $\|Z u(t, \cdot)\|_{H^{\sigma}}$ for some $\sigma$ satisfying $1 \ll \sigma \ll s$, and in $(B),\left(B^{\prime}\right),\|u(t, \cdot)\|_{L^{\infty}}$ by $\|u(t, \cdot)\|_{C^{\rho}}$ for some $\rho$ with $1 \ll \rho \ll s$. This does not bring any essential new difficulty.

- In the statement of Theorem 2, we were assuming that the initial data admitted the action of a large number of iterates of $\left(x \partial_{x}\right)$, which would correspond in the model $(32)$ above to make act a large number of vector fields $Z$. This was due to the fact that in $[6$ some non optimal choice was made in the proof of $L^{\infty}$ estimates. On the other hand, in the work of Ionescu and Pusateri [35] and of Ifrim and Tataru [32, only one vector field has to be used. Below, inspired by $[32,30$, we shall adopt an optimal point of view that allows one to use only one vector field in the analysis of model (32), following the method of 6 .

Proposition 4 implies immediately the global existence result in Theorem 2 when combined with local existence theory.

The fact that $(A)$ and $(B)$ imply $\left(A^{\prime}\right)$ is essentially trivial for the model equation 32 . Actually, writing the energy inequality for $(32)$ and $(35)$, one gets

$$
\begin{aligned}
\|u(t, \cdot)\|_{H^{s}}+\|Z u(t, \cdot)\|_{L^{2}} & \leq C\left[\|u(1, \cdot)\|_{H^{s}}+\|Z u(1, \cdot)\|_{L^{2}}\right. \\
& \left.+\int_{1}^{t}\left[\|N(u(\tau, \cdot))\|_{H^{s}}+\|Z N(u(\tau, \cdot))\|_{L^{2}}\right] d \tau\right] .
\end{aligned}
$$


As $N$ is cubic in $(u, \bar{u})$, the right hand side of $(38)$ is bounded from above by (39)

$$
C\left[\|u(1, \cdot)\|_{H^{s}}+\|Z u(1, \cdot)\|_{L^{2}}+\int_{1}^{t}\|u(\tau, \cdot)\|_{L^{\infty}}^{2}\left[\|u(\tau, \cdot)\|_{H^{s}}+\|Z u(\tau, \cdot)\|_{L^{2}}\right] d \tau\right] .
$$

Plugging (36) into $(39)$, choosing $A$ large enough and then $\epsilon_{0}$ small enough in function of $A, B$, one deduces estimate $\left(A^{\prime}\right)$.

Of course, in the case of system (9) (with $d=1$, infinite depth and $\kappa=0$ ), the proof of the corresponding inequality is much more technical, as one has to cope with the difficulties explained in sections 2 and 4 in the case of Sobolev energy inequalities. Estimates in $L^{2}$ for $Z u$ instead of $u$ are performed in a similar way, using the new unknown $\omega$ and a normal form in order to get rid of the quadratic part of the nonlinearity.

The remaining step, in order to complete the proof of Theorem 2 is to show that $\left(B^{\prime}\right)$ holds for solutions of the equation under assumptions $(A)$ and $(B)$.

\section{Optimal decay estimates}

The key point in order to prove the enhanced decay estimate $\left(B^{\prime}\right)$ from $(A)$ and $(B)$, both in the case of the simplified model $(32)$ or for the true water waves equation, is to derive from the PDE an ODE whose analysis will provide the wanted $L^{\infty}$ bounds, as well as the asymptotics of the solution.

Several approaches have been used by different authors in order to do so. Ionescu and Pusateri [35] work in Fourier space in order to get an ODE for the Fourier transform of the solution. Ifrim and Tataru 32 use a wave packets description of the solution, for which they obtain an ODE, working thus in phase-space variables. The approach in Alazard-Delort [6] relies on the rewriting of the PDE under study in a semi-classical framework, with a Planck constant $h=\frac{1}{t}$, so that the ODE one looks for is obtained as the semi-classical limit of the quantum problem provided by the PDE. This is the method we present below, blending the approach of [6] (which was not optimal regarding to phasespace decomposition) with some of the ideas of Ifrim and Tataru [32, 30]. Let us introduce:

Definition 5 Let $\delta \in\left[0, \frac{1}{2}\right], m \in \mathbb{R}$. We denote by $S_{\delta}^{m}(\mathbb{R} \times \mathbb{R})$ the space of smooth functions $(h, x, \xi) \rightarrow a_{h}(x, \xi)$, defined for $h$ in $\left.] 0,1\right],(x, \xi)$ in $\mathbb{R} \times \mathbb{R}$, satisfying estimates

$$
\left|\left(h \partial_{h}\right)^{\gamma} \partial_{x}^{\alpha} \partial_{\xi}^{\beta} a_{h}(x, \xi)\right| \leq C h^{-\delta(\alpha+\beta)}\langle\xi\rangle^{m} .
$$

If $a$ is in $S_{\delta}^{m}(\mathbb{R} \times \mathbb{R})$, we define its Weyl-quantization by the formula

$$
\mathrm{Op}_{h}^{\mathrm{W}}(a) v=\frac{1}{2 \pi h} \int e^{i(x-y) \frac{\xi}{h}} a\left(\frac{x+y}{2}, \xi\right) v(y) d y d \xi
$$

for $v$ in $\mathcal{S}(\mathbb{R})$. We denote by $H_{\mathrm{sc}}^{s}(\mathbb{R})$ the space of families of functions $v=$ $\left(v_{h}\right)_{h \in] 0,1]}$ such that, if we define

$$
\left\|v_{h}\right\|_{H_{h}^{s}}=\left\|\mathrm{Op}_{h}^{\mathrm{W}}\left(\langle\xi\rangle^{s}\right) v_{h}\right\|_{L^{2}}=\left\|\left\langle h D_{x}\right\rangle^{s} v_{h}\right\|_{L^{2}},
$$


one has

$$
\|v\|_{H_{\mathrm{sc}}^{s}} \stackrel{\text { def }}{=} \sup _{h \in] 0,1]}\left\|v_{h}\right\|_{H_{h}^{s}}<+\infty .
$$

Then $\mathrm{Op}_{h}^{\mathrm{W}}(a)$ acts from $H_{\mathrm{sc}}^{s}$ to $H_{\mathrm{sc}}^{s-m}$. Consider now a solution $u$ to equation (32) and define a new function $v(t, x)$, related to $u$ through

$$
u(t, x)=\frac{1}{\sqrt{t}} v\left(t, \frac{x}{t}\right) .
$$

Set $h=\frac{1}{t}$. Then $v$ solves the equation

$$
\left(D_{t}-\mathrm{Op}_{h}^{\mathrm{W}}(x \xi+p(\xi))\right) v=h\left[\alpha_{3} v^{3}+\alpha_{1}|v|^{2} v+\alpha_{-1}|v|^{2} \bar{v}+\alpha_{-3} \bar{v}^{3}\right] .
$$

Let us introduce the set

$$
\Lambda=\left\{(x, \xi) \in \mathbb{R}^{2} ; x+p^{\prime}(\xi)=0\right\} .
$$

The basic idea is that this set carries the most important part of the solution, so that one may deduce an ODE from (44) restricting the symbol $x \xi+p(\xi)$ to $\Lambda$, and showing that the error one generates in that way decays like an integrable power of $t$ when $t$ goes to infinity.

A key point is that, in the case $p(\xi)=|\xi|^{\frac{1}{2}}$ corresponding to the linearized water waves in our model $(32),, \Lambda$ is a graph: there is a smooth function $\varphi$ : $\mathbb{R}^{*} \rightarrow \mathbb{R}$, given by $\varphi(x)=-\frac{1}{4|x|}$ such that

$$
\Lambda=\left\{(x, \xi) \in \mathbb{R}^{2} ; \xi=d \varphi(x)\right\} .
$$

We shall ignore in the rest of this discussion the technicalities related to the behaviour of $\varphi$ at zero or infinity and shall do like if $v$ where spectrally supported on a compact subset of $\mathbb{R}-\{0\}$, i.e. we shall assume (abusively) that $v=$ $\mathrm{Op}_{h}^{\mathrm{W}}(\chi(\xi)) v$ for some $\chi$ in $C_{0}^{\infty}(\mathbb{R}-\{0\})$. We take next $\gamma$ in $C_{0}^{\infty}(\mathbb{R})$, equal to one close to zero, and define, inspired by Ifrim and Tataru [30],

$$
v_{\Lambda}=\mathrm{Op}_{h}^{\mathrm{W}}\left(\gamma\left(\frac{x+p^{\prime}(\xi)}{\sqrt{h}}\right)\right) v, v_{\Lambda^{c}}=v-v_{\Lambda}
$$

where the choice of the width $\sqrt{h}$ in the cut-off function is the optimal one. Then our aim is to get for $v_{\Lambda}$ an ordinary differential equation.

Proposition 6 Let $v$ be a solution of (44). Assume that for $t$ in some interval $[1, T]$ the a priori estimates (36) hold true. Then, if we define $\omega(x)=x d \varphi(x)+$ $p(d \varphi(x)), v_{\Lambda}$ solves

$$
\begin{aligned}
\left(D_{t}-\omega(x)\right) v_{\Lambda}=h\left[\alpha_{3} v_{\Lambda}{ }^{3}+\alpha_{1}\left|v_{\Lambda}\right|^{2} v_{\Lambda}+\alpha_{-1}\left|v_{\Lambda}\right|^{2} \bar{v}_{\Lambda}\right. & \left.+\alpha_{-3} \bar{v}_{\Lambda}^{3}\right] \\
& +O_{L^{\infty}}\left(\epsilon h^{1+\delta}\right),
\end{aligned}
$$

where $\delta$ is a small positive number. 
Idea of proof: The proof of the proposition relies on the following facts. First, the contribution $v_{\Lambda^{c}}$ defined in 47 will have better estimates than $v$ : this follows from the fact that by definition

$$
v_{\Lambda^{c}}=\mathrm{Op}_{h}^{\mathrm{W}}\left(\gamma_{1}\left(\frac{x+p^{\prime}(\xi)}{\sqrt{h}}\right) \frac{x+p^{\prime}(\xi)}{\sqrt{h}}\right) v \simeq \mathrm{Op}_{h}^{\mathrm{W}}\left(\gamma_{1}\left(\frac{x+p^{\prime}(\xi)}{\sqrt{h}}\right)\right)(\sqrt{h} \mathcal{L} v)
$$

where $\gamma_{1}(z)=\frac{(1-\gamma(z))}{z}$ and $\mathcal{L}=\frac{1}{h} \mathrm{Op}_{h}^{\mathrm{W}}\left(x+p^{\prime}(\xi)\right)$. It turns out that $\mathcal{L}$ may be expressed from the Klainerman vector field $Z$ and the equation, so that an a priori bound of the form $(A)$ in $(36)$ implies that

$$
\|\mathcal{L} v\|_{L^{2}}=O\left(h^{-K \epsilon^{2}}\right)
$$

and thus $\left\|v_{\Lambda^{c}}\right\|_{L^{2}}=O\left(h^{\frac{1}{2}-K \epsilon^{2}}\right)$. As the cut-off $\gamma_{1}$ in 49 localizes essentially in a strip of frequencies of size $h^{\frac{1}{2}}$, a semiclassical Sobolev inequality provides estimates of the form $\left\|v_{\Lambda^{c}}\right\|_{L^{\infty}}=O\left(\epsilon h^{\frac{1}{4}-K \epsilon^{2}}\right)$. This allows to replace in the right hand side of (44) $v$ by $v_{\Lambda}$, up to contributions to the remainder. One may also perform such a replacement in the left hand side using some commutation arguments, ending up with an equation of the form

$$
\begin{aligned}
& \left(D_{t}-\mathrm{Op}_{h}^{\mathrm{W}}(x \xi+p(\xi)) v_{\Lambda}\right. \\
& \quad=h\left[\alpha_{3} v_{\Lambda}^{3}+\alpha_{1}\left|v_{\Lambda}\right|^{2} v_{\Lambda}+\alpha_{-1}\left|v_{\Lambda}\right|^{2} \bar{v}_{\Lambda}+\alpha_{-3} \bar{v}_{\Lambda}^{3}\right]+O\left(\epsilon h^{\frac{5}{4}-\delta}\right) .
\end{aligned}
$$

Finally, as $\frac{d}{d \xi}(x \xi+p(\xi))=x+p^{\prime}(\xi)$ vanishes on $\Lambda$, and as this set may be represented using (46), one may write through a Taylor expansion at $\xi=d \varphi(x)$,

$$
x \xi+p(\xi)=x d \varphi(x)+p(d \varphi(x))+O\left((\xi-d \varphi(x))^{2}\right) .
$$

Since we have restricted our considerations to the case of $\xi$ staying in a compact subset of $\mathbb{R}^{*}$ (which is equivalent to $x$ staying in a compact subset of $\mathbb{R}^{*}$ when $(x, \xi)$ is close to $\Lambda)$, one may rewrite this as

$$
x \xi+p(\xi)=\omega(x)+O\left(\left(x+p^{\prime}(\xi)\right)^{2}\right) .
$$

Recalling the definition (47) of $v_{\Lambda}$, we deduce from that

$$
\begin{aligned}
\mathrm{Op}_{h}^{\mathrm{W}}(x \xi+p(\xi)) v_{\Lambda} & =\omega(x) v_{\Lambda} \\
+ & \text { term in } \mathrm{Op}_{h}^{\mathrm{W}}\left(\left(x+p^{\prime}(\xi)\right)^{2}\right) \mathrm{Op}_{h}^{\mathrm{W}}\left(\gamma\left(\frac{x+p^{\prime}(\xi)}{\sqrt{h}}\right)\right) v
\end{aligned}
$$

The last term above may be written, up to remainders, as

$$
\sqrt{h} \mathrm{Op}_{h}^{\mathrm{W}}\left(\frac{x+p^{\prime}(\xi)}{\sqrt{h}} \gamma\left(\frac{x+p^{\prime}(\xi)}{\sqrt{h}}\right)\right) \underbrace{\mathrm{Op}_{h}^{\mathrm{W}}\left(x+p^{\prime}(\xi)\right) v}_{=h \mathcal{L} v} .
$$

Combining with (50), and the fact that the localization of $v_{\Lambda}$ allows one to estimate $L^{\infty}$ norms from $h^{-\frac{1}{4}}$ times Sobolev ones, we obtain that the $L^{\infty}$ norm 
of 54 is $O\left(\epsilon h^{\frac{5}{4}-\delta}\right)$ for some small $\delta>0$, so that the last term in $(53)$ may be incorporated in the remainder (48). Plugging (53) in (51), one gets (48).

End of proof of Theorem 2. As explained at the end of section 5, to conclude the proof of the theorem, one has to show that, for a solution $u$ of $(33)$, the a priori bounds (36) imply (37). We have already seen that that $\left(A^{\prime}\right)$ holds and we are left with showing the $L^{\infty}$ estimate $\left(B^{\prime}\right)$. As we have seen that $v_{\Lambda^{c}}$ enjoys good a priori bounds, one has to obtain a uniform bound for the solution $v_{\Lambda}$ of the ODE 48. Performing a normal form, one may reduce 48 to an equation

$$
\left(D_{t}-\omega(x)\right) f=h \alpha_{1}|f|^{2} f+O_{L^{\infty}}\left(\epsilon h^{1+\delta}\right), h=\frac{1}{t},
$$

where $f$ is a new unknown related to $v_{\Lambda}$ in such a way that a uniform control of $f$ is equivalent to a uniform control of $v_{\Lambda}$. As we assumed that $\alpha_{1}$ in $(33)$ is real, if there were no remainder in $\epsilon t^{-1-\delta}$ in $(55)$, one would get immediately that $\partial_{t}|f|^{2}=0$, whence the uniform control of $f$. Since the $O\left(\epsilon t^{-1-\delta}\right)$ remainder in 55 is integrable, one may show that such a uniform control still holds for the solution of 55 . If one makes this reasoning keeping track of the dependence of the constants on $A, B$, one may prove that $\left(B^{\prime}\right)$ follows from $(A),(B)$. Moreover, the analysis of the ODE provides as well the asymptotics of the solution when $t$ goes to $+\infty$.

This outline of proof concerns the simplified model (32). In the case of the water waves system, the general strategy of the last part of the proof is similar. The fact that the coefficient $\alpha_{1}$ in $(33)$ is real (that plays a crucial role above) is a "null condition" in the sense of Christodoulou-Klainerman, that holds true because of the structure of the water waves system.

\section{$7 \quad$ Further results}

Our goal in this section is to discuss further results of long time existence, concerning equation (9) under different assumptions.

We consider first the case of initial data that decay in space. We have discussed up to now, for such data, equation (9) when $g>0, \kappa=0$ and the depth of the fluid is infinite. We give here references to other global existence results, under other assumptions.

Water waves in infinite depth. We consider gravity waves $(g>0, \kappa=0)$ in infinte depth, as in Theorem 2 In that statement, and in the results of Ionescu-Pusateri 35] and Ifrim and Tataru 32, the assumptions of smallness of the initial data involve norms that control the energy. It turns out that one may weaken these conditions: Wang proved in [50, a global existence result for the gravity water waves equation in one space dimension, and for infinite depth fluids, when the initial data $\left(\eta,\left|D_{x}\right|^{\frac{1}{2}} \psi\right)$ belongs to some homogeneous Sobolev space, that contains functions with infinite energy. 
Capillary and capillary-gravity waves in infinite depth. Consider first equation (9) with $g=0$ and $\kappa>0$, still for a two dimensional fluid of infinite depth, (i.e. $x$ in $(9)$ varies in $\mathbb{R}$ ). For small, smooth and decaying initial data, global existence has been proved independently by Ifrim and Tataru 33 and by Ionescu and Pusateri [34]. As far as we know, no (almost) global existence result is known for solutions of the full capillary-gravity problem ( 9 with $g>0, \kappa>0)$ in infinite depth, when the space dimension is equal to one. On the other hand, the similar problem in two space dimensions (i.e. for three dimensional fluids) has been solved by Deng, Ionescu, Pausader and Pusateri. They proved global existence for small smooth decaying data in [24].

Water waves in finite depth. Much less results are known concerning global existence of solutions when one works with a fluid of finite depth. The only results we are aware of concern the case of two space dimensions (three dimensional fluids) with $g>0, \kappa=0$ or $g=0, \kappa>0$ in (9). Wang studied [53, 51, 52] the existence of global solutions for small, smooth, decaying initial data.

To finish this section, let us also discuss related results of long time existence, when one considers small but not necessarily decaying initial data. In this case, one cannot expect to use dispersion in order to get a longer existence time than the one that holds in general for a quadratic non linear hyperbolic equation with small data of size $\epsilon$, namely $T_{\epsilon} \sim \epsilon^{-1}$. Nevertheless, we have seen in section 4 that a normal forms procedure may allow one to reduce essentially the equation to a cubic one, and so allows to expect an existence time $T_{\epsilon}$ bounded from below by $c \epsilon^{-2}$.

For fluids of infinite depth, such a property has been proved in the case of gravity waves $(g>0, \kappa=0)$ by Totz and Wu 49 in one space dimension and by Totz [48] in two space dimensions. In the case of capilarity waves ( $g=$ $0, \kappa>0)$ in one space dimension, a similar result has been obtained by Ifrim and Tataru [33] and by Ionescu and Pusateri [34]. When one considers a constant non zero vorticity, a lower bound in $c \epsilon^{-2}$ for the time of existence of solutions has been shown by Ifrim and Tataru [31, still in one space dimension.

Regarding finite depth fluids, Harrop-Griffith, Ifrim and Tataru [27] have proved a $c \epsilon^{-2}$ lower bound for the existence time, in the gravity waves case $(g>0, \kappa=0)$ in one space dimension.

The above results apply in particular when one considers initial data that are periodic in space, i.e. defined on the circle. In such a case, better results may be obtained under stronger assumptions. First, it is possible to construct special classes of global solutions. Actually, Plotnikov and Toland [43] (resp. Iooss, Plotnikov and Toland [36]) constructed, for the gravity waves system in finite (resp. infinite) depth, standing waves solutions. For the full gravity-capilarity system in infinite depth, Alazard and Baldi [1] did the same. Later, Berti and Montalto [15] built up time quasi-periodic solutions of system (9) in infinite depth, and more recently Baldi, Berti, Haus and Montalto [13] treated the same problem in finite depth.

The preceding results do not concern the Cauchy problem, as one constructs special solutions. But combining some of the ideas of [1] and normal forms 
methods, Berti and Delort [14] proved that system (9), with even periodic initial

data of size $\epsilon$, has solutions defined up to time $c_{N} \epsilon^{-N}$ for any $N$, when the parameters $(g, \kappa)$ avoid an exceptional subset of zero measure.

\section{References}

[1] T. Alazard and P. Baldi. Gravity capillary standing water waves. Arch. Ration. Mech. Anal., 217(3):741-830, 2015.

[2] T. Alazard, N. Burq, and C. Zuily. On the water-wave equations with surface tension. Duke Math. J., 158(3):413-499, 2011.

[3] T. Alazard, N. Burq, and C. Zuily. Strichartz estimates for water waves. Ann. Sci. Éc. Norm. Supér. (4), 44(5):855-903, 2011.

[4] T. Alazard, N. Burq, and C. Zuily. On the Cauchy problem for gravity water waves. Invent. Math., 198(1):71-163, 2014.

[5] T. Alazard, N. Burq, and C. Zuily. Cauchy theory for the gravity water waves system with non-localized initial data. Ann. Inst. H. Poincaré Anal. Non Linéaire, 33(2):337-395, 2016.

[6] T. Alazard and J.-M. Delort. Global solutions and asymptotic behavior for two dimensional gravity water waves. Ann. Sci. Éc. Norm. Supér. (4), 48(5):1149-1238, 2015.

[7] T. Alazard and J.-M. Delort. Sobolev estimates for two dimensional gravity water waves. Astérisque, (374):viii+241, 2015.

[8] T. Alazard and G. Métivier. Paralinearization of the Dirichlet to Neumann operator, and regularity of three-dimensional water waves. Comm. Partial Differential Equations, 34(10-12):1632-1704, 2009.

[9] S. Alinhac. Paracomposition et opérateurs paradifférentiels. Comm. Partial Differential Equations, 11(1):87-121, 1986.

[10] S. Alinhac. Ondes de raréfaction pour des systèmes quasi-linéaires hyperboliques multidimensionnels. In Journées "Équations aux Dérivées Partielles" (Saint Jean de Monts, 1988), pages Exp. No. VIII, 7. École Polytech., Palaiseau, 1988.

[11] D. M. Ambrose. Well-posedness of vortex sheets with surface tension. SIAM J. Math. Anal., 35(1):211-244, 2003.

[12] D. M. Ambrose and N. Masmoudi. The zero surface tension limit of twodimensional water waves. Comm. Pure Appl. Math., 58(10):1287-1315, 2005 .

[13] P. Baldi, M. Berti, E. Haus, and R. Montalto. Time quasi-periodic gravity water waves in finite depth. Preprint, 2017. 
[14] M. Berti and J.-M. Delort. Almost global existence of solutions for capillary-gravity water waves equations with periodic spatial boundary conditions. Preprint, 2017.

[15] M. Berti and R. Montalto. KAM for gravity capillary water waves. Memoires of $A M S, 2017$.

[16] J.-M. Bony. Calcul symbolique et propagation des singularités pour les équations aux dérivées partielles non linéaires. Ann. Sci. École Norm. Sup. (4), 14(2):209-246, 1981.

[17] D. Christodoulou and H. Lindblad. On the motion of the free surface of a liquid. Comm. Pure Appl. Math., 53(12):1536-1602, 2000.

[18] D. Coutand and S. Shkoller. Well-posedness of the free-surface incompressible Euler equations with or without surface tension. J. Amer. Math. Soc., 20(3):829-930, 2007.

[19] W. Craig. An existence theory for water waves and the Boussinesq and Korteweg-de Vries scaling limits. Comm. Partial Differential Equations, 10(8):787-1003, 1985.

[20] W. Craig and C. Sulem. Numerical simulation of gravity waves. J. Comput. Phys., 108(1):73-83, 1993.

[21] J.-M. Delort. Long-time Sobolev stability for small solutions of quasilinear Klein-Gordon equations on the circle. Trans. Amer. Math. Soc., 361(8):4299-4365, 2009.

[22] J.-M. Delort. A quasi-linear Birkhoff normal forms method. Application to the quasi-linear Klein-Gordon equation on $\mathbb{S}^{1}$. Astérisque, (341):vi+113, 2012.

[23] J.-M. Delort. Quasi-linear perturbations of Hamiltonian Klein-Gordon equations on spheres. Mem. Amer. Math. Soc., 234(1103):vi+80, 2015.

[24] Y. Deng, A. D. Ionescu, B. Pausader, and F. Pusateri. Global solutions of the gravity-capilarity water waves system in 3 dimensions. Preprint, 2015.

[25] P. Germain, N. Masmoudi, and J. Shatah. Global solutions for 2D quadratic Schrödinger equations. J. Math. Pures Appl. (9), 97(5):505-543, 2012.

[26] P. Germain, N. Masmoudi, and J. Shatah. Global solutions for the gravity water waves equation in dimension 3. Ann. of Math. (2), 175(2):691-754, 2012 .

[27] B. Harrop-Griffiths, M. Ifrim, and D. Tataru. Finite depth gravity water waves in holomorphic coordinates. Ann. PDE, 3(1):Art. 4, 102, 2017.

[28] J. K. Hunter, M. Ifrim, and D. Tataru. Two dimensional water waves in holomorphic coordinates. Comm. Math. Phys., 346(2):483-552, 2016. 
[29] J. K. Hunter, M. Ifrim, D. Tataru, and T. K. Wong. Long time solutions for a Burgers-Hilbert equation via a modified energy method. Proc. Amer. Math. Soc., 143(8):3407-3412, 2015.

[30] M. Ifrim and D. Tataru. Global bounds for the cubic nonlinear Schrödinger equation (NLS) in one space dimension. Nonlinearity, 28(8):2661-2675, 2015 .

[31] M. Ifrim and D. Tataru. Two dimensional gravity water waves with constant vorticity: I. cubic lifespan. Preprint, 2015.

[32] M. Ifrim and D. Tataru. Two dimensional water waves in holomorphic coordinates II: Global solutions. Bull. Soc. Math. France, 144(2):369-394, 2016 .

[33] M. Ifrim and D. Tataru. The Lifespan of Small Data Solutions in Two Dimensional Capillary Water Waves. Arch. Ration. Mech. Anal., 225(3):12791346, 2017.

[34] A. D. Ionescu and F. Pusateri. Global regularity for $2 d$ water waves with surface tension. Preprint, 2015.

[35] A. D. Ionescu and F. Pusateri. Global solutions for the gravity water waves system in 2d. Invent. Math., 199(3):653-804, 2015.

[36] G. Iooss, P. I. Plotnikov, and J. F. Toland. Standing waves on an infinitely deep perfect fluid under gravity. Arch. Ration. Mech. Anal., 177(3):367478, 2005.

[37] D. Lannes. Well-posedness of the water-waves equations. J. Amer. Math. Soc., 18(3):605-654, 2005.

[38] D. Lannes. Space time resonances [after Germain, Masmoudi, Shatah]. Astérisque, (352):Exp. No. 1053, ix, 355-388, 2013. Séminaire Bourbaki. Vol. 2011/2012. Exposés 1043-1058.

[39] D. Lannes. The water waves problem, volume 188 of Mathematical Surveys and Monographs. American Mathematical Society, Providence, RI, 2013. Mathematical analysis and asymptotics.

[40] H. Lindblad. Well-posedness for the motion of an incompressible liquid with free surface boundary. Ann. of Math. (2), 162(1):109-194, 2005.

[41] V. I. Nalimov. The Cauchy-Poisson problem. Dinamika Splošn. Sredy, (Vyp. 18 Dinamika Židkost. so Svobod. Granicami):104-210, 254, 1974.

[42] T. Ozawa, K. Tsutaya, and Y. Tsutsumi. Remarks on the Klein-Gordon equation with quadratic nonlinearity in two space dimensions. In Nonlinear waves (Sapporo, 1995), volume 10 of GAKUTO Internat. Ser. Math. Sci. Appl., pages 383-392. Gakkōtosho, Tokyo, 1997. 
[43] P. I. Plotnikov and J. F. Toland. Nash-Moser theory for standing water waves. Arch. Ration. Mech. Anal., 159(1):1-83, 2001.

[44] J. Shatah. Normal forms and quadratic nonlinear Klein-Gordon equations. Comm. Pure Appl. Math., 38(5):685-696, 1985.

[45] J. Shatah and C. Zeng. Geometry and a priori estimates for free boundary problems of the Euler equation. Comm. Pure Appl. Math., 61(5):698-744, 2008.

[46] J. Shatah and C. Zeng. A priori estimates for fluid interface problems. Comm. Pure Appl. Math., 61(6):848-876, 2008.

[47] J. Shatah and C. Zeng. Local well-posedness for fluid interface problems. Arch. Ration. Mech. Anal., 199(2):653-705, 2011.

[48] N. Totz. A justification of the modulation approximation to the $3 \mathrm{D}$ full water wave problem. Comm. Math. Phys., 335(1):369-443, 2015.

[49] N. Totz and S. Wu. A rigorous justification of the modulation approximation to the 2D full water wave problem. Comm. Math. Phys., 310(3):817$883,2012$.

[50] X. Wang. Global infinite energy solutions for the $2 d$ gravity water waves system. Preprint, 2015.

[51] X. Wang. Global solutions for the $3 d$ water waves system above a flat bottom. Preprint, 2015.

[52] X. Wang. Global regularity for the $3 d$ finite depth capillary water waves. Preprint, 2017.

[53] X. Wang. On the 3-dimensional gravity water waves system above a flat bottom. Anal. PDE, 10(4):893-928, 2017.

[54] S. Wu. Well-posedness in Sobolev spaces of the full water wave problem in 2-D. Invent. Math., 130(1):39-72, 1997.

[55] S. Wu. Well-posedness in Sobolev spaces of the full water wave problem in 3-D. J. Amer. Math. Soc., 12(2):445-495, 1999.

[56] S. Wu. Almost global wellposedness of the 2-D full water wave problem. Invent. Math., 177(1):45-135, 2009.

[57] S. Wu. Global wellposedness of the 3-D full water wave problem. Invent. Math., 184(1):125-220, 2011.

[58] H. Yosihara. Gravity waves on the free surface of an incompressible perfect fluid of finite depth. Publ. Res. Inst. Math. Sci., 18(1):49-96, 1982.

[59] V. E. Zakharov. Stability of periodic waves of finite amplitude on the surface of a deep fluid. Journal of Applied Mechanics and Technical Physics, 9(2):190-1943, 1968. 